scheme embodies in it a certain amount of contract practice, in that the staff of the hospitals receive 25 per cent. of the sum collected. We think it extremely undesirable that the character of the institutions should be thus changed and that the staff who give their services voluntarily should become paid servants of the hospital, and we are wondering if the staffs of the three great hospitals above-mentioned realize the conditions which the adoption of this scheme entails.

\title{
Medical Advice to the Public
}

Medical Men who give gratuitous advice to the public should be very careful as to the accuracy of their statements. In a recent publication on "Venereal Diseases and their Effects," by the National Council for Combating Venereal Diseases, there is a paragraph on the "Effect of Gonorrhoea on Offspring." It is there stated that " a child born to a woman suffering from gonorrhoea runs a grave risk; during birth, of receiving the infection in the. eyes. The result is the development a few days after birth of an acute inflammation of the eyes (ophthalmia neonatorum), which is a very frequent source of complete blindness." The writer goes on to state, that "This disease can be prevented if drops of 1 per cent. protargol are instilled into the child's eyes immediately after birth." As it stands this statement is grossly inaccurate, and even if there has been a printer's error in the strength of the solution, the statement is open to grave doubt. That prophylactic treatment with drugs has proved beneficial has been shown by Crede's treatment-in which, as a matter of fact, 1 per cent. silver nitrate was used. The routine use of such methods, however, by midwives and others, is liable to cause a false sense of security (which would certainly be increased by the above statement), and is furthermore liable to misuse and serious mistakes. Thus, we have heard of a case in which strong nitric acid was dropped into a baby's eye in mistake for a silver preparation.

In any case, seeing that presumably these pamphlets of the National Council are not circulated among medical men only, we regard it as highly undesirable that any instructions as to treatment with drugs should be given. It would have been wiser to have laid stress upon the supreme importance of immediately referring cases of this nature to treatment by competent medical men. 